\title{
CORRESPONDENCE
}

\section{Physical Assessment by Pharmacists: A Valued Component of Care}

I read with interest the study by Chevalier and colleagues in the November-December 2016 issue of the Canadian Journal of Hospital Pharmacy. ${ }^{1}$ Briefly, the authors administered a survey on expectations for various domains of pharmaceutical care to the nurses and physicians of a surgical ward before and 8 months after implementation of clinical pharmacy services. I was intrigued by the low proportion of respondents from each profession who felt that "conducting basic physical assessments" was an expected role for a clinical pharmacist on the surgical ward. Here, I elaborate on possible explanations for these results, and share some of my own experiences as a clinical pharmacist incorporating physical assessment on a surgical ward.

Before implementation of clinical pharmacy services in the study reported by Chevalier and colleagues, ${ }^{1} 12 \%$ and $0 \%$ of nurse and physician respondents, respectively, agreed that conducting basic physical assessment was an expected role of clinical pharmacists on surgical wards; these proportions remained virtually unchanged, at $11 \%$ and $0 \%$, respectively, in the followup survey. Given the many physical manifestations of adverse drug events, these results contradict the high proportions of respondents who expected pharmacists to identify adverse drug reactions. ${ }^{1}$ Indeed, based on countless discussions with nurses and physicians about pharmacist-performed physical assessment, I would suggest that the vague description of "basic" physical assessment in this survey likely resulted in a substantial proportion of respondents unfamiliar with the concept interpreting it as "pharmacists making medical diagnoses". However, the purpose of pharmacistperformed physical assessment is to complement the rest of the clinical assessment in identifying and monitoring for the resolution of drug-related problems. There is variation among pharmacists in the complexity of physical assessment performed in their practice, but every clinical pharmacist performs an inspection, the most "basic" form of physical assessment, every time they meet and engage in discussion with a patient, identifying findings relevant to drug therapy, such as discomfort, altered mental status, and conspicuous rashes. With various techniques becoming standard in entry-to-practice pharmacy education, we must be diligent in clearly defining the role and scope of pharmacist-performed physical assessment.

I offer details about my use of physical assessment in a neurosurgical ward to illustrate that pharmacist-performed physical assessment can improve patient care in a surgical ward, and can come to be highly valued by nurses and physicians. I took on the role of clinical pharmacist for the neurosurgical service of a quaternary care hospital in 2015 after completing my residency and PharmD training, which incorporated didactic and hands-on education related to physical assessment. I immediately began looking for ways to incorporate physical assessment into my practice. At first, nurses and (particularly) physicians were skeptical, but they soon accepted the role of physical assessment in my overall assessments, especially after hearing my brief explanation as to how I was incorporating it into my pharmaceutical care plans, and seeing it in action.

Over the course of about 3 months, I observed the processes of care on the neurosurgical ward and identified key disease states and drugs that would benefit from pharmacist-performed physical assessment. For example, in settings where I had previously practised, such as an internal medicine clinical teaching unit, medical staff routinely performed comprehensive volume assessments in inpatients with heart failure or cirrhosis with ascites, such that I could easily interpret these data and integrate them into my recommendations. However, I noticed that for the many patients admitted to neurosurgery for intracranial hemorrhage who had concomitant heart failure, this information was not readily available. Because I had the training to do so, I began performing comprehensive volume assessments, including examination of the jugular venous pulsation, heart, and lungs, in these patients to collect and document these data. Other notable examples included monitoring for resolution of postoperative complications, such as pneumonia and wound infections, and identifying the physical manifestations of antipsychotic-induced oculogyric crisis in a patient previously presumed to have focal seizures.

In all cases, I collaborated with my nurse and physician colleagues in enacting a pharmaceutical care plan to resolve these significant drug-related problems. As I fostered interprofessional relationships with the rest of the neurosurgical team, physical 
assessment naturally became a valued — and, indeed, expectedcomponent of the care that I delivered. In light of this experience, I believe we should consider other professionals' expectations and opinions about our role, but we should never let them dissuade us from practising to our full scope. After all, before the 1960s, no one expected to see pharmacists working on the wards.

Reference

1. Chevalier B, Neville HL, Thompson K, Nodwell L, MacNeil M. Health care professionals' opinions and expectations of clinical pharmacy services on a surgical ward. Can J Hosp Pharm. 2016;69(6):439-48.

Ricky D Turgeon, BScPharm, ACPR, PharmD

Department of Medicine, Division of Cardiology

University of Alberta

Edmonton, Alberta

Competing interests: None declared. 